\title{
ENHANCEMENT OF SUPERCRITICAL FLUID EXTRACTION IN MEMBRANE CLEANING PROCESS BY ADDITION OF ORGANIC SOLVENTS
}

\author{
Jan Krzysztoforski, Andrzej Krasiński, Marek Henczka, Wojciech Piątkiewicz \\ Warsaw University of Technology, Faculty of Chemical and Process Engineering, Waryńskiego 1, \\ 00-645 Warsaw, Poland
}

\begin{abstract}
In this study, the process of membrane cleaning by supercritical fluid extraction was investigated. Polypropylene microfiltration membranes, contaminated with oils, were treated in a batch process with a supercritical fluid (SCF). As extractant, pure supercritical carbon dioxide or supercritical carbon dioxide with admixtures of methanol, ethanol and isopropanol were used. Single-stage and multi-stage extraction was carried out and process efficiency was determined. The obtained results showed that addition of organic solvents significantly enhances the cleaning performance, which increases with increase of organic solvent concentration and decreases with increasing temperature. All three solvents showed a comparable effect of efficiency enhancement. The results confirmed that supercritical fluid extraction can be applied for polypropylene membrane cleaning.
\end{abstract}

Keywords: supercritical fluid, extraction, porous membrane, TIPS

\section{INTRODUCTION}

Microporous polypropylene membranes are used in a variety of industrial applications including separation processes (e.g. microfiltration, diafiltration, etc.), gas-liquid contactors and supported liquid membranes. Recently, they are also widely applied as a support for composite membranes for gas separation (Szwast, 2012). Polypropylene membranes are produced by many different techniques, among which the Thermally Induced Phase Separation (TIPS) is thought to be one of the most effective ones. Application of the TIPS method enables effective process control with high reproducibility of the final microstructure of produced membranes. It can be applied to a wide range of polymers. The TIPS process starts with dissolving a melted polymer in a diluent of high boiling point, typically oil of low molecular mass, at high temperature $\left(170-200{ }^{\circ} \mathrm{C}\right)$. The resulting mixture is cast or extruded into the desired shape, usually a flat panel or hollow fibre, and then cooled down to induce phase separation and polymer solidification (Szwast et al., 2008). The microstructure of solidified polymer can be controlled by the cooling rate, solution composition and cooling liquid composition. After the solidification of polymer is completed, the resulted membrane must be cleaned to remove the diluent remaining in pores. Usually for this purpose the physical extraction process using organic solvents is applied (Berghmans et al., 1996). Organic solvents are also used in maintenance operations in order to remove contaminations accumulated during normal membrane usage. The substantial disadvantage of the application of volatile compounds is that during their evaporation the membrane may swell or contract due to local pressure changes at the pore walls. Moreover, the cleaning efficiency of conventional organic solvent method is limited by the rate of molecular mass transfer between the diluent and the extractant. In addition to the mentioned disadvantages, the use of organic solvents in membrane 
treatment makes the process hazardous, environmentally unfriendly and significantly expensive due to high solvent regeneration costs.

Recently, supercritical fluids (SCFs) have been found as promising media that may replace organic solvents in membrane production, maintenance and modification technologies. Most properties of SCFs are very sensitive to small variations of pressure and temperature, rendering SCFs easily tunable with respect to their applicability as solvents or extractants. Nevertheless, the dissolving power of a SCF may only approach that of an organic solvent at high densities, so the solubility of a solute in SCFs is usually smaller than that in organic solvents. An interesting phenomenon, especially for mass transfer through porous systems, is that the surface tension of a liquid in equilibrium with its vapour decreases with increasing temperature and becomes zero at the critical point. Therefore, the properties of SCF are similar to gaseous compounds, so that it uniformly fills up the available space, which enables to penetrate readily into porous solids and packed beds (Baiker and Wandeler, 2000). The combination of liquid-like and gas-like properties together with good control of physicochemical properties, as well as excellent penetrability, make SCF a remarkable substance for membrane processing.

In industrial applications, supercritical carbon dioxide $\left(\mathrm{scCO}_{2}\right)$ is the most popular SCF used as reaction and extraction medium due to its nontoxicity, non-flammability, relatively low critical parameters $\left(T_{\mathrm{k}}=304.1 \mathrm{~K}, p_{\mathrm{k}}=7.38 \mathrm{MPa}\right)$ and low cost. All these features make $\mathrm{scCO}_{2}$ consistent with principles of Green Chemistry. Supercritical carbon dioxide has been successfully applied in various processes, both as a solvent and reaction medium (Bogel-Łukasik et al., 2009; Brunner 2004, 2010; Conceiao et al., 2012; Sovova and Stateva, 2011; Yin et al., 2005). Examples of current $\mathrm{scCO}_{2}$ applications in membrane technologies are presented in literature (Akin and Temelli, 2011; Li et al., 2008; Xinli et al., 2009; Zhang et al., 2007). It was experimentally proved that the extraction process carried out using $\mathrm{scCO}_{2}$ as carrier fluid is very effective due to its unique properties. Using this method, membranes with low levels of residual contaminants may be obtained, which is critical in some sophisticated applications to meet the end-users' needs. In comparison to the extraction performed using organic solvent, the application of $\mathrm{scCO}_{2}$ possesses an important advantage - it allows to clean and dry the polymer membrane rapidly without shrinkage and collapsing of its internal structure due to absence of vapour-liquid interactions in pores. This behaviour is of high importance for preserving the primary microstructure of the membrane. Moreover, the diluent dissolved in $\mathrm{scCO}_{2}$ can be easily separated from the gaseous $\mathrm{scCO}_{2}$ after mixture depressurisation and then recycled to the process. Stepwise depressurisation can potentially enable fractionation of mixtures. Nevertheless, in some specific cases impact of $\mathrm{scCO}_{2}$ may cause unfavourable changes in solid material structure and properties. In literature, the effects of supercritical carbon dioxide on polypropylene have been reported (Lei et al., 2007). The authors observed solubility of $\mathrm{scCO}_{2}$ in amorphous polypropylene and accompanying swelling of its structure. According to their experimental results and computations both of these effects first decrease and then increase with temperature, reaching the minimum at $100{ }^{\circ} \mathrm{C}$ for pressure up to 100 bar. At higher pressure large uncertainties exist for $\mathrm{CO}_{2}$ solubility due to unpredictable changes in PP crystallinity, which are coupled to swelling degree. In other work the influence of chemical treatment on structure and strength parameters of polypropylene hollow fibre membranes was studied (Szwast et al., 2007). Washing of membranes with isopropanol and $5 \%$ solution of hydrochloric acid was recommended by authors as it leads to consistent values of porosity and mechanical properties. In addition, the treatment with $5 \% \mathrm{HCl}$ resulted in slight modification of pore size distribution towards smaller sizes. The pore size distribution was recalculated from bubble pressure test, so one can conclude that the observed effect is due to more efficient extraction of impurities from tight pores using this solvent. However, it has to be confirmed for any particular material that cleaning process does not negatively affect structural or mechanical properties of membranes. The main difficulty of application of $\mathrm{scCO}_{2}$ for extraction of high boiling substance from polypropylene membrane is relatively low solubility of diluent in extractant. In this paper the methods to improve the final efficiency of the process by addition of a small amount of organic solvent to $\mathrm{scCO}_{2}$ are proposed. This process is experimentally investigated by adding methanol $(\mathrm{MeOH})$, ethanol $(\mathrm{EtOH})$ and isopropanol $(\mathrm{iPrOH})$ as 
solubility enhancers applied in membrane extraction process. In the second part of the paper, effects of supercritical carbon dioxide on mechanical properties of porous polypropylene membranes are discussed.

\section{EXPERIMENTAL}

\subsection{Materials and equipment}

The membranes used in the experiments were commercially available ACCUREL ${ }^{\circledR}$ hydrophobic capillary membranes Type PP S6/2 used for microfiltration, manufactured by Membrana GmbH, Germany. These membranes are produced using the TIPS method. The membrane parameters are as follows: wall thickness $450 \pm 50 \mu \mathrm{m}$, inner diameter $1800 \pm 50 \mu \mathrm{m}$, length approx. $1.3 \mathrm{~m}$, porosity ca. $70 \%$ (estimation based on determined apparent density of the membranes). Membranes of this type are used in bundles of 600 membranes in microfiltration modules. The membranes were contaminated with a soybean oil/castor oil mixture, which can be applied in TIPS process for manufacturing such type of membranes. Three types of organic admixtures were alternatively used in the proposed method, namely methanol, ethanol and isopropanol. Isopropanol is often used as pure organic solvent in the conventional TIPS method for removal of oil residues after phase separation. In order to determine the influence of alcohol type on cleaning efficiency, two other alcohols, methanol and ethanol, which belong to the same homologous series as isopropanol, were studied in this work as well. In Table 1, materials used in the experimental investigations are listed.

Table 1. Materials used in the experiments

\begin{tabular}{|l|c|c|}
\hline \multicolumn{1}{|c|}{ Material } & Purity & Source \\
\hline Carbon dioxide & $99.9992 \%$ & Linde Gaz Polska Sp. z o.o., Poland \\
\hline Helium & $99.995 \%$ & Linde Gaz Polska Sp. z o.o., Poland \\
\hline Methanol & $99.8 \%$ & Chempur, Poland \\
\hline Ethanol & $99.8 \%$ & Chempur, Poland \\
\hline Isopropanol & $99.0 \%$ & Stanlab, Poland \\
\hline Soybean oil & food grade & pharmacy \\
\hline Castor oil & food grade & pharmacy \\
\hline
\end{tabular}

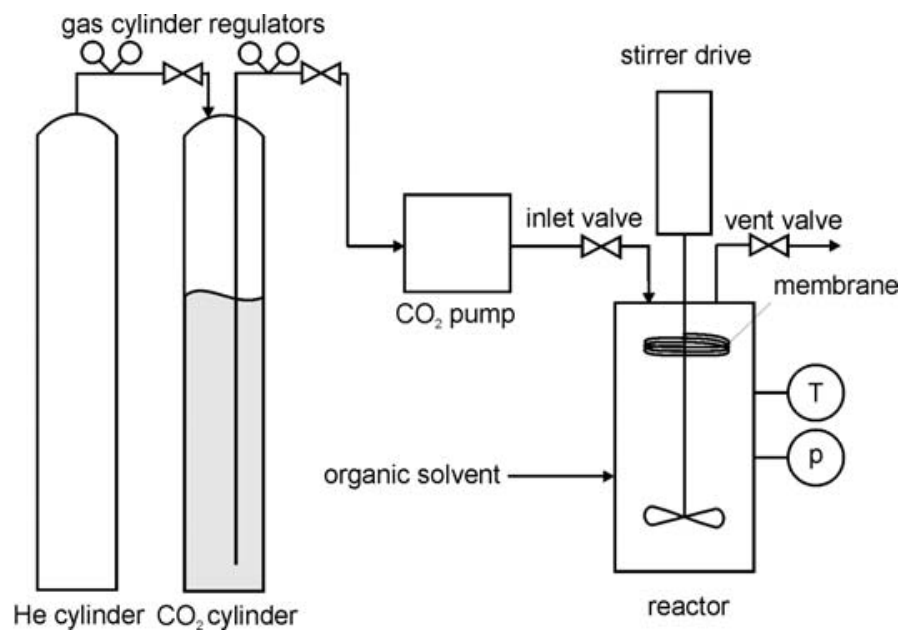

Fig. 1. Experimental setup 
In Figure 1, a scheme of the experimental system is presented. Carbon dioxide was supplied from a high pressure cylinder equipped with a dip tube. An additional cylinder containing helium was connected to the $\mathrm{CO}_{2}$ cylinder in order to elevate the $\mathrm{CO}_{2}$ pressure in the cylinder to the desired level. Carbon dioxide in liquid state flowed to the $\mathrm{CO}_{2}$ pump (SFT-10 $\mathrm{CO}_{2}$ Pump by Supercritical Fluid Technologies, Inc., USA). The pump pressurised $\mathrm{CO}_{2}$, which was subsequently fed to the process vessel (High Pressure Reactor by PARR, Inc., USA; volume: $1200 \mathrm{~mL}$ ). The reactor was equipped with a stirrer, a temperature control system, a pressure gauge and process valves. In Figure 1, the position of the membrane in the stirred tank during the experiments is schematically shown.

\subsection{Supercritical fluid extraction}

In the first part of the experiments, supercritical fluid extraction of contaminants from membranes at different process conditions in a batch mode was carried out. The procedure for all performed experiments was as follows. A clean membrane was weighed on an analytical scale (accuracy \pm 0.0001 g). The membrane was immersed in a soybean oil/castor oil mixture (2:1 by volume) for $24 \mathrm{~h}$. Then, the membrane was taken out and hung up for $24 \mathrm{~h}$ in order to remove the excessive oil left in the membrane. After this procedure, the remaining oil was present in the membrane pores only. The resulted membrane was weighed once again, and the initial mass of oil in raw material was determined. Subsequently, the contaminated membrane was placed in the high pressure reactor in a coiled form (see Figure 1). In the case of organic solvent addition, an adequate volume of organic solvent was added to the reactor. Then the reactor was closed and purged for 5 min with $\mathrm{CO}_{2}$ at ambient pressure in order to remove air from the reactor. Then $\mathrm{CO}_{2}$ was fed into the reactor and the desired process conditions were achieved by adjustment of the $\mathrm{CO}_{2}$ pump and the temperature control system. During the extraction all parameters were kept constant and the reactor content was agitated to intensify the process. After a specific time, the reactor was decompressed and the membrane was removed from the reactor. In the case of experiments with addition of an organic solvent, the membrane was dried for $24 \mathrm{~h}$ at ambient temperature in order to enable evaporation of the organic solvent remaining in the pores. Finally, the mass of the membrane after extraction was determined in order to estimate the oil mass loss.

The extraction efficiency $\eta$ was calculated using the following equation:

$$
\eta=\frac{\Delta m}{m} \cdot 100 \%
$$

where $m$ is the initial oil mass in a raw membrane and $\Delta m$ is the mass loss of oil resulting from the extraction process.

Three types of supercritical fluid extraction experiments were carried out:

1. Single-stage extraction. The procedure described above was followed once for each membrane.

2. Multi-stage extraction. The procedure described above was followed three times for each membrane. The purified membrane was placed in the reactor again, and the extraction process was repeated. The membrane was treated in each stage with pure $\mathrm{scCO}_{2}$ or a mixture of $\mathrm{scCO}_{2}$ with an organic solvent.

3. Process rate determination. Experiments were carried out at given conditions as single-stage processes for different extraction times.

Experimental conditions for the performed experiments are summarised in Table 2. 
Table 2. Conditions of experimental investigations

\begin{tabular}{|l|c|c|c|}
\hline \multirow{2}{*}{} & \multicolumn{3}{|c|}{ Experiment type } \\
\cline { 2 - 4 } & Single-stage & Multi-stage & Process rate \\
\hline Pressure $[\mathrm{MPa}]$ & 18 & 18 & 18 \\
\hline Agitation speed $[\mathrm{rpm}]$ & 300 & 300 & 300 \\
\hline Temperature $\left[{ }^{\circ} \mathrm{C}\right]$ & $40,70,100$ & 70 & 70 \\
\hline Time $[\mathrm{min}]$ & 120 & 120 & $5,30,60,120$ \\
\hline \multirow{3}{*}{ Organic solvent type } & $\mathrm{MeOH}$ & $\mathrm{MeOH}$ & EtOH \\
\cline { 2 - 4 } & $\mathrm{EtOH}$ & $\mathrm{EtOH}$ & \\
\cline { 2 - 4 } & $\mathrm{iPrOH}$ & $\mathrm{iPrOH}$ & \\
\hline Organic solvent concentration $[\mathrm{mL} / \mathrm{L}]$ & $0,50,100$ & 0,100 & 100 \\
\hline
\end{tabular}

For the experimental conditions, the solubility of soybean oil in pure supercritical carbon dioxide was determined using an empirical correlation proposed and fitted to experimental data by Jokić et al. (2011), which enables to calculate solubility $S$ of soybean oil as a function of $\operatorname{scCO}_{2}$ density $\rho$ and temperature $T\left(a_{1}, a_{2}\right.$, and $a_{3}$ are constants):

$$
S=\rho^{\left(a_{1}+a_{2} \rho\right)} \exp \left(\frac{a_{3}}{T}\right)
$$

For $18 \mathrm{MPa}$ and $313 \mathrm{~K}, 343 \mathrm{~K}$ and $373 \mathrm{~K}$, the solubility values are $3.3 \mathrm{~g} / \mathrm{L}, 1.9 \mathrm{~g} / \mathrm{L}$, and $0.9 \mathrm{~g} / \mathrm{L}$, respectively (calculated for the volume of carbon dioxide in process conditions).

\section{RESULTS AND DISCUSSION}

\subsection{Single-stage extraction}

In Table 3, the results of extraction efficiency for the single-stage process are summarised. The results are also plotted in Figures 2-5. The influence of organic solvent addition is distinct, as in all conditions the process efficiency for mixtures of $\mathrm{scCO}_{2}$ with organic solvent was much higher than that for pure $\mathrm{scCO}_{2}$. One can observe two clear tendencies when analysing the experimental results. The extraction efficiency increases with the increase of organic solvent content. The effect of solubility enhancement induced by addition of organic solvents depends on their concentration and is higher for higher solvent concentrations. Application of any considered organic solvent used in the experiments showed comparable influence effect with regard to the process enhancement. Another trend is that in most cases extraction efficiency decreases with increasing temperature. However, due to complex features of three-component supercritical systems, this is not a general rule. This dependence can be explained by the decrease of $\mathrm{scCO}_{2}$ density with the increase of temperature at constant pressure. Solubility of substances in SCF is higher, when the SCF density is higher. The high value of extraction efficiency for one experiment $\left(100 \mathrm{~mL} / \mathrm{L} \mathrm{MeOH}\right.$ at $\left.100{ }^{\circ} \mathrm{C}\right)$, which does not match the described effect of temperature, can be explained by the formation of a two-phase system, as the methanol-carbon dioxide system has a miscibility gap at these conditions. The intention was that the solvent should be present as one phase, so conditions as in the experiment variant described above should be avoided for representative comparison. Moreover, it would not be appropriate for possible implementation of the new cleaning method, as the method is based on the fact that the cleaning fluid is in supercritical state, not in liquid state. Even if the cleaning efficiency for this variant is higher than that for all other 
presented variants, the liquid cleaning fluid would be difficult to recover - as in the existing method utilising pure organic solvent cleaning fluids.

Table 3. Results of single-stage extraction efficiency $\eta$

\begin{tabular}{|c|c|c|c|c|}
\hline \multirow{2}{*}{$\begin{array}{c}\text { Temperature } \\
{\left[{ }^{\circ} \mathrm{C}\right]}\end{array}$} & $\begin{array}{c}\text { Organic solvent concentration } \\
{[\mathrm{mL} / \mathrm{L}]}\end{array}$ & $\mathrm{MeOH}$ & $\mathrm{EtOH}$ & iPrOH \\
\cline { 2 - 5 } & 0 & 19.54 & 19.54 & 19.54 \\
\cline { 2 - 5 } 40 & 50 & 36.58 & 28.09 & 31.98 \\
\cline { 2 - 5 } & 100 & 80.88 & 91.13 & 68.20 \\
\hline \multirow{3}{*}{70} & 0 & 15.15 & 15.15 & 15.15 \\
\cline { 2 - 5 } & 50 & 30.57 & 44.99 & 33.23 \\
\cline { 2 - 5 } & 100 & 56.17 & 69.76 & 61.78 \\
\hline \multirow{3}{*}{100} & 0 & 9.82 & 9.82 & 9.82 \\
\cline { 2 - 5 } & 50 & 15.30 & 32.86 & 41.61 \\
\cline { 2 - 5 } & 100 & 99.64 & 62.94 & 46.82 \\
\hline
\end{tabular}

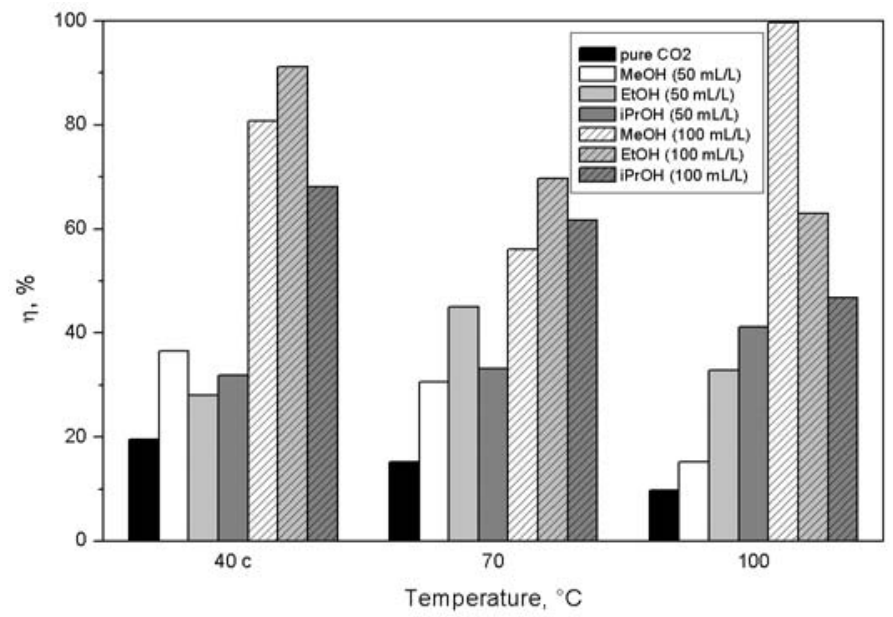

Fig. 2. The effect of temperature on single-stage extraction efficiency $\eta$

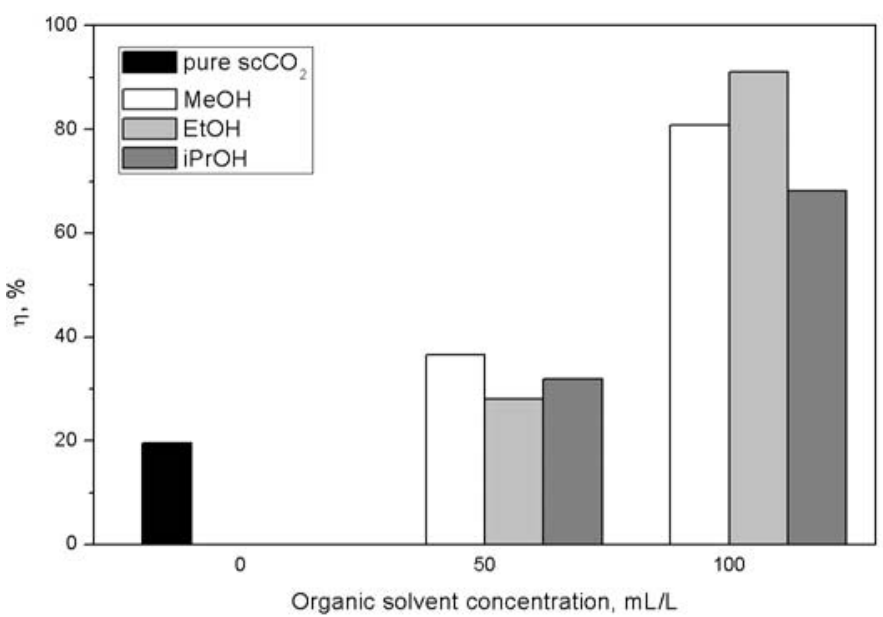

Fig. 3. The effect of organic solvent concentration on single-stage extraction efficiency $\eta$ at $40{ }^{\circ} \mathrm{C}$ 


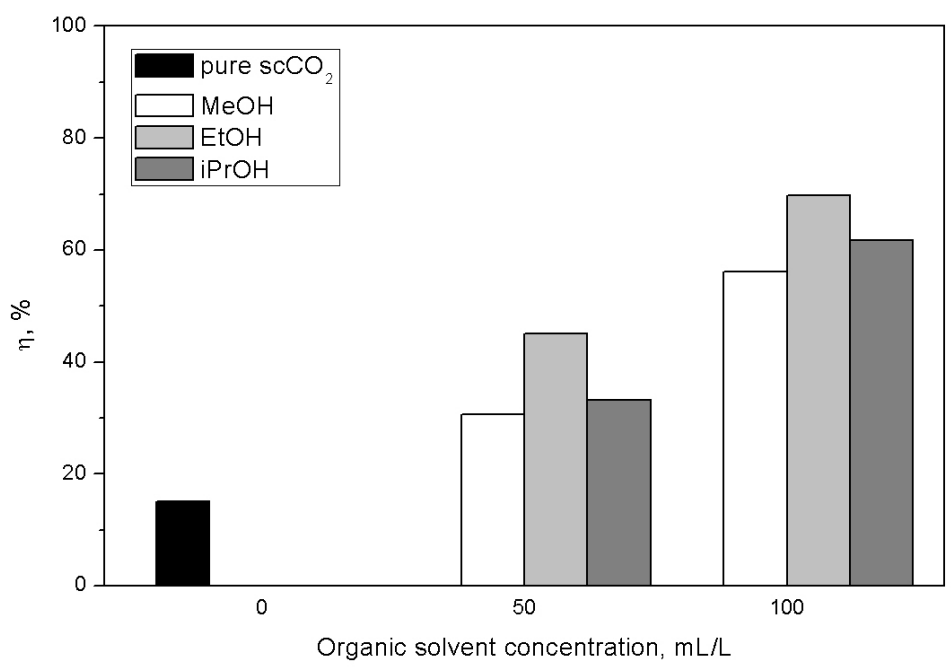

Fig. 4. The effect of organic solvent concentration on single-stage extraction efficiency $\eta$ at $70{ }^{\circ} \mathrm{C}$

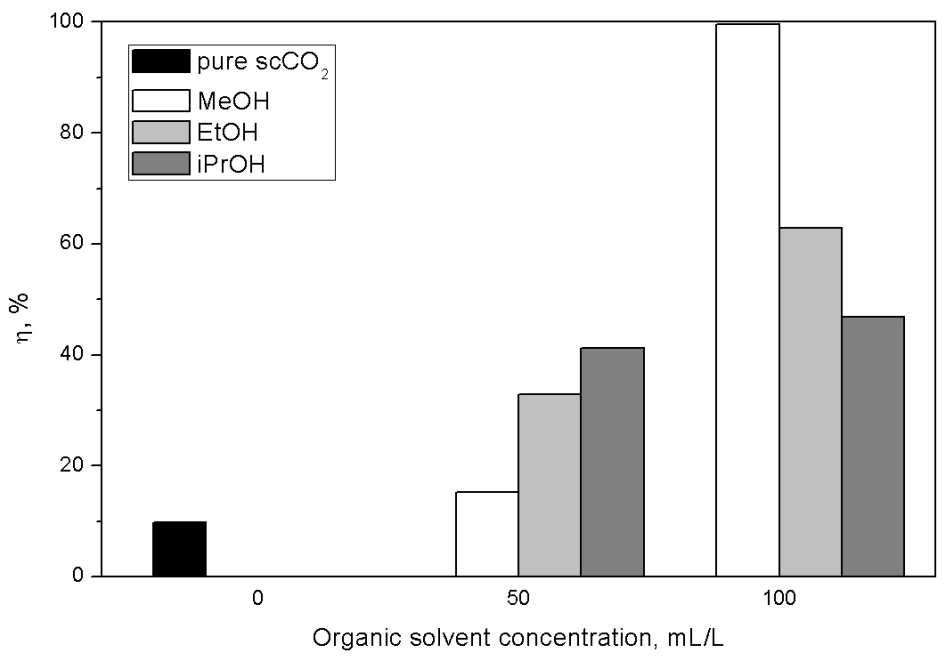

Fig. 5. The effect of organic solvent concentration on single-stage extraction efficiency $\eta$ at $100{ }^{\circ} \mathrm{C}$

\subsection{Multi-stage extraction}

As the second type of experiments, multi-stage extraction was carried out. Contaminated membranes were treated with $\mathrm{scCO}_{2}$ three times. The results for multi-stage experiments are summarised in Table 4 , and they are also plotted in Figure 6 . The process conditions were set according to Table 2. One can see that in a 3-stage process, much higher extraction efficiency can be achieved, when compared to the single stage process. The best results were obtained for ethanol, for which membrane cleaning efficiency exceeded $99 \%$.

Table 4. Results of multi-stage extraction efficiency, $\eta$

\begin{tabular}{|c|c|c|c|c|}
\hline \multirow{2}{*}{ Stage } & \multicolumn{4}{|c|}{$\eta[\%]$} \\
\cline { 2 - 5 } & $\mathrm{CO}_{2}$ & $\mathrm{MeOH}$ & $\mathrm{EtOH}$ & iPrOH \\
\hline 1 & 15.15 & 56.17 & 69.76 & 61.78 \\
\hline 2 & 18.21 & 91.71 & 95.32 & 87.96 \\
\hline 3 & 19.39 & 96.79 & 99.19 & 93.45 \\
\hline
\end{tabular}




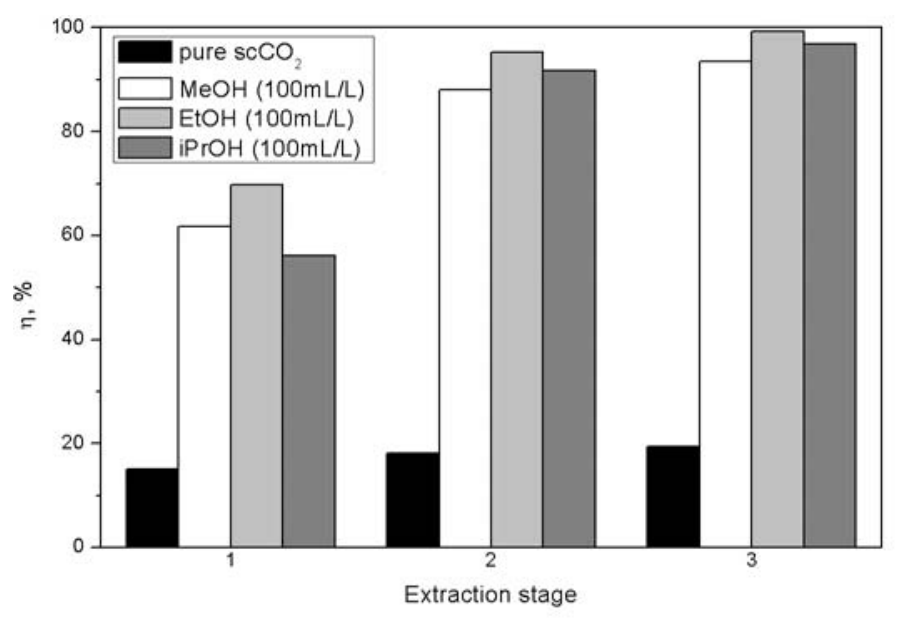

Fig. 6. Multi-stage extraction efficiency $\left(70^{\circ} \mathrm{C}, 18 \mathrm{MPa}, 120 \mathrm{~min}, 100 \mathrm{~mL} / \mathrm{L}\right.$ organic solvent)

\subsection{Process rate determination}

From the practical point of view, another important factor besides the efficiency is time that is necessary to achieve high performance of cleaning. For this purpose, the process rate was investigated. The extraction time directly determines the equipment sizing for given throughput, hence it reflects process economy. The experiments were carried out using a $\mathrm{scCO}_{2} / \mathrm{EtOH}$ mixture, as it showed to be the best cleaning fluid in terms of efficiency. The process conditions were set according to Table 2 . The obtained results are summarised in Table 5 and presented in Figure 7. One can see that after 120 min of extraction process, the system asymptotically reached the equilibrium state, which defines the upper limit for the cleaning efficiency of the membrane.

Table 5. The increase of extraction efficiency in time

\begin{tabular}{|c|c|}
\hline Process time $[\mathrm{min}]$ & $\eta[\%]$ \\
\hline 0 & 0.00 \\
\hline 5 & 52.71 \\
\hline 30 & 63.27 \\
\hline 60 & 68.03 \\
\hline 120 & 69.76 \\
\hline
\end{tabular}

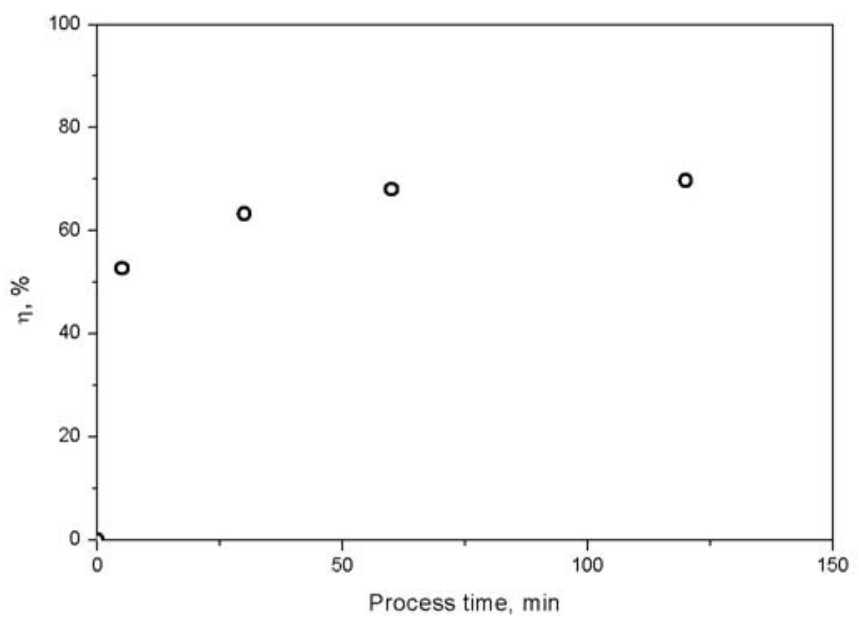

Fig. 7. Extraction efficiency $\eta$ vs. process time $\left(70{ }^{\circ} \mathrm{C}, 18 \mathrm{MPa}\right)$ 


\subsection{Discussion}

The experiments showed that addition of small amounts of organic solvents to supercritical carbon dioxide leads to an increase of the extraction efficiency. Generally, the observed effect is stronger for higher organic solvent concentrations and lower temperatures. In the experiments, tubular porous polypropylene membranes were used, but other microporous membranes can be cleaned using this method as well. The process presented in this paper can be applied not only to substances remaining in membrane pores after phase separation during the TIPS process, but in general to all liquid contaminations present in membrane pores, especially to substances having a low solubility in pure supercritical carbon dioxide.

An important aspect of the method is the proper choice of the organic solvent. As can be noticed, all three organic solvents used in the experiments (methanol, ethanol, isopropanol) showed a similar effect in terms of enhancement of the cleaning process. However, in industrial applications other factors have to be taken into account, i.e. costs, health and safety (H\&S) considerations. From this perspective, isopropanol seems to be the most economic choice as well as the least problematic in terms of handling, which directly reflects the process safety. Of course, other suitable organic solvents, which are known as solubility enhancers in $\mathrm{scCO}_{2}$, can also be used.

The amount of residual alcohol in pores was not directly determined by any analytical method. However, the experimental results show clearly that for the 3-stage experiments, initial mass of membranes was almost regained. It means that only a small amount of remaining contaminant was present in pores. Due to lower solubility and higher viscosity of oil mixture it is more likely that nonextracted residues consist of oil rather than alcohols. In addition, for the pore size of microfiltration membranes alcohol molecules escape the porous structure easily and spontaneously. Such a mass loss was observed in experimental studies. And this is why after treatment the membranes were left for 24 hours for evaporation. However, organic solvents are also present in the pores after the traditional TIPS method (at even higher concentrations, as pure organic solvents are used), so this feature of the presented method does not constitute a drawback with regard to the state of the art. In general, the presence of small amounts of organic solvent does not limit the applicability of microporous membranes, as they normally undergo a special ageing process (the last stage of membrane production), during which residual solvents evaporate and leave membrane pores. For medical applications, additional cleaning measures are employed. Moreover, in the presented method the amount of residual solvent present in the pores after cleaning can be reduced further by using pure $\mathrm{scCO}_{2}$, which will be explained in detail below.

In this study, single-stage and multi-stage extraction processes in a batch system were carried out. Single-stage batch extraction has a limited efficiency due to solubility limitations. In the experimental system used in this study oil remains in the extraction vessel and it can cause secondary contamination of the membrane after depressurisation. A multi-stage batch process resulted in higher extraction efficiency values, although it does not overcome all the limitations of the single-stage batch process. However, the process can also be conducted in a continuous flow apparatus. In this case, a membrane (or a bunch of membranes) is fixed in a tubular vessel and supercritical carbon dioxide containing an organic solvent admixture flows through the vessel. The main advantage of this concept is cost reduction due to potential $\mathrm{scCO}_{2}$ and organics recovery and recirculation back to the process. The control of the system is easier, but the capital investment related to the equipment is slightly higher. A properly designed system should enable reasonable hydrodynamics not only on the outer surface of the membranes, but also inside the lumina. Unlike in the batch process, in the continuous flow mode oil is removed from the surroundings of the membrane and from the extraction vessel during the process. On the other hand, typical solutions known in supercritical fluid technologies can be applied, which further improves the process. After extraction, the supercritical medium containing carbon dioxide, organic solvent and oil, can be depressurised (below the critical point), after which carbon dioxide is present as 
a separate phase. $\mathrm{CO}_{2}$ can be easily recycled. Depressurisation can be also carried out in more than one stage. After the first stage (lower pressure than in the extraction vessel, but still above the critical point), the solubility of oil is much lower than that in the extraction conditions and it leads to condensation of a separate oil phase, which can be separated and removed from the system. However, in these conditions the organic solvent still remains in the supercritical fluid phase. After the second depressurisation stage (below the critical point), the organic solvent is separated from carbon dioxide. In this way, the organic solvent can be recovered and used many times like $\mathrm{CO}_{2}$, which leads to a very low consumption of both organic solvent and carbon dioxide. Moreover, the organic solvent concentration can vary in time, e.g. can be higher at the beginning of the extraction process and can gradually decrease, which helps to minimise the organic solvent amount present in the extraction vessel after depressurisation.

When a supercritical fluid is applied to a cleaning process, one has to be aware of its impact on purified materials. Hence, it has to be confirmed that the process does not negatively affect the structural properties of membranes which could change filtration parameters such as selectivity and efficiency. The influence of supercritical carbon dioxide on the structure and mechanical properties of porous polypropylene membranes was investigated in a previous study (Krzysztoforski et al., 2012). In that work, clean polypropylene membranes (the same as used in the present work, but without oil present in pores) were treated with pure supercritical carbon dioxide at identical conditions as in the present study (process time $2 \mathrm{~h}$, temperature 40,70 , and $100{ }^{\circ} \mathrm{C}$, pressure $18 \mathrm{MPa}$ ). These experiments were conducted in order to examine a possible destruction of membrane structure during $\mathrm{scCO}_{2}$ treatment, which would disqualify the membranes for further application. After $\mathrm{scCO}_{2}$ treatment, the tensile test experiment, bubble point test and analysis of SEM microphotographs were performed. A membrane not treated with $\mathrm{scCO}_{2}$ was considered as the reference sample. No change nor destruction of membrane morphology could be clearly confirmed based on the SEM microphotographs. The tensile test results revealed that $\mathrm{scCO}_{2}$ treatment resulted in a decrease of the Young's modulus by $36-48 \%$ and the ultimate tensile strength by approx. 7-8\% (stronger effect for higher temperatures). However, these changes do not limit the normal use of the membranes, as there is still a considerable safety margin in normal microfiltration process conditions. The results of bubble point investigations showed that $\mathrm{scCO}_{2}$ treatment causes an increase of the number of pores and a slight increase of the filtration coefficient value as well, which in turn has a positive effect on the course of microfiltration using treated membranes. Moreover, the pore size distributions and mean pore sizes remained practically unchanged. An extended description of the experimental methods as well as detailed results of these experiments can be found in (Krzysztoforski et al., 2012). Although $\mathrm{scCO}_{2}$ penetration in membrane materials is the main cause for changes in polymer properties and no critical deterioration could be observed for the experiments with pure $\mathrm{scCO}_{2}$, it is not apparent that the same will apply to membrane treatment using $\mathrm{scCO}_{2}$-organic solvent systems. Hence, further investigations have to be carried out on this issue. From the results described above, one can discuss the influence of the novel cleaning procedure on permeability and selectivity of the membranes. The ultrafiltration coefficient UFC was not changed significantly. As UFC represents the membrane permeability, one can conclude that permeability was not affected by the process. In the case of microfiltration membranes the separation mechanism is based on simple classification by size, where cut off diameter corresponds to a pore size. Therefore, no significant change in pore size distributions will affect the selectivity of the membranes. This was confirmed in bubble pressure test, where reference membranes were compared with treated ones for different process conditions. No significant changes of the pore size distribution were observed. Therefore, one can conclude that operational parameters of the membrane were not affected significantly by the treatment procedure. Lower process temperatures are preferred due to higher process efficiency and weaker changes of membrane properties.

The investigations will be continued using recently produced membranes containing oil from the TIPS method, instead of artificially contaminated membranes. A more accurate method for determination of the real amount of residuals in the membrane (e.g. TOC analysis) will be used in future research as 
complement of the weight-base method. The process will be investigated in a continuous flow system and experiments with the use of pure organic solvents will be carried out for comparison purposes. Moreover, the membrane structure and performance changes caused by supercritical extraction with and without organic admixtures will be investigated in more detail.

\section{CONCLUSIONS}

In this study, the cleaning process of tubular polypropylene membranes by supercritical fluid extraction using pure $\mathrm{scCO}_{2}$ or mixtures of $\mathrm{scCO}_{2}$ with organic solvents was investigated. It has been experimentally confirmed that addition of organic solvents to $\mathrm{scCO}_{2}$ is favourable as it significantly increases the process efficiency. Using a mixture of $\mathrm{scCO}_{2}$ and an organic solvent, a smaller amount of $\mathrm{scCO}_{2}$ is necessary to efficiently extract and dissolve the contaminant in the bulk fluid. The cleaning efficiency increases with organic solvent concentration due to an increase of contaminant solubility induced by organic solvent admixture. All three organic solvents used in the experiments (methanol, ethanol, isopropanol) showed a similar effect in terms of enhancement of the cleaning process, although isopropanol seems to be the most economic choice as well as the least problematic in terms of handling. The cleaning efficiency decreases with increasing temperature, which can be explained by the decrease of supercritical fluid density, that negatively affects the dissolving power of the supercritical fluid. Effects of supercritical carbon dioxide on membrane structure and mechanical properties were also considered. Treatment of polypropylene membranes with $\mathrm{scCO}_{2}$ is not destructive for them and does not limit the application of treated membranes in typical microfiltration processes (Krzysztoforski et al., 2012). The presented method enables to clean membranes during their production or membrane regeneration after normal usage. Addition of organic solvents helps to achieve the same cleaning efficiency at lower pressures as in the case of application of pure supercritical carbon dioxide. The method also meets the principles of Green Chemistry, which postulates limitation of organic solvent usage, especially in comparison to the conventional method utilising pure organic solvents. In the presented method, organics are used in smaller amounts and they can be easily recovered and recycled back to the process. Investigation of the process in a continuous flow mode and further research on membrane structure and properties changes caused by treatment with $\mathrm{scCO}_{2}$ will be the subject of future work.

The research was supported by scientific funds in 2010-2013 as a scientific project (N N209 175 238). It was also partially supported by the State Grant (N R05 0051 06).

\section{SYMBOLS}

$\begin{array}{ll}a_{1}, a_{2}, \text { and } a_{3} & \text { constants } \\ m & \text { initial oil mass in a membrane, } \mathrm{g} \\ S & \text { solubility, } \mathrm{g} / \mathrm{L} \\ T & \text { temperature, } \mathrm{K} \\ \Delta m & \text { oil mass loss, } \mathrm{g}\end{array}$

Greek symbols

$\eta \quad$ cleaning efficiency, $\%$

$\rho \quad$ density, $\mathrm{g} / \mathrm{L}$ 


\section{REFERENCES}

Akin O., Temelli F., 2011. Effect of supercritical $\mathrm{CO}_{2}$ flux, temperature and processing time on physicochemical and morphological properties of commercial reverse osmosis membranes. J. Supercrit. Fluids, 60, 81 - 88. DOI: 10.1016/j.supflu.2011.03.011.

Baiker A., Wandeler R., 2000. Supercritical fluids; opportunities in heterogeneous catalysis. CATTECH 4, 128143. DOI: $10.1023 / \mathrm{A}: 1011943227355$.

Berghmans S., Berghmans H., Meijer H., 1996. Spinning of hollow porous fibres via the TIPS mechanism. J. Membrane Sci., 116, 171 -189. DOI: 10.1016/0376-7388(96)00037-3.

Bogel-Łukasik E., Gomes Da Silva M., Nogueira I. D., Bogel-Łukasik R., Nunes Da Ponte M., 2009. Study on selectivity of myrcene hydrogenation in high-pressure carbon dioxide catalysed by noble metal catalysts. Green Chem., 11, 1847-1856. DOI: 10.1039/B916017P.

Brunner G., 2004. Supercritical Fluids as Solvents and Reaction Media. Elsevier.

Brunner G., 2010. Applications of supercritical fluids. Annu. Rev. Chem. Biomol. Eng., 1, 321-342. DOI: 10.1146/annurev-chembioeng-073009-101311.

Conceiao L., Bogel-Łukasik R., Bogel-Łukasik E., 2012. Supercritical $\mathrm{CO}_{2}$ as an effective medium for a novel conversion of glycerol and alcohols in the heterogeneous telomerisation of butadiene. Green Chem., 14, 673-681. DOI: 10.1039/C2GC16149D.

Jokić S., Svilović S., Zeković Z., Vidović S., 2011. Mathematical modelling of soybean oil solubility in supercritical carbon dioxide. Int. J. Food Sci. Technol., 46, 1031-1037. DOI: 10.1111/j.13652621.2011.02571.x.

Krzysztoforski J., Krasiński A., Henczka M., Piątkiewicz W., Szwast M., 2012. Influence of supercritical carbon dioxide on structure and mechanical properties of porous polypropylene membranes. Challenges of Modern Technol., 3, $20-25$.

Lei Z., Ohyabu H., Sato Y., Inomata H., Smith Jr. R.L., 2007. Solubility, swelling degree and crystallinity of carbon dioxide-polypropylene system. J. Supercrit. Fluids 40, 452 - 461. DOI: 10.1016/j.supflu.2006.07.016.

Li Z., Tang H., Liu X., Xia Y., Jiang J., 2008. Preparation and characterization of microporous poly(vinyl butyral) membranes by supercritical $\mathrm{CO}_{2}$-induced phase separation. J. Membrane Sci., 312, 115 - 124. DOI: 10.1016/j.memsci.2007.12.043.

Sovova H., Stateva R.P., 2011. Supercritical fluid extraction from vegetable materials. Rev. Chem. Eng., 27, 79156. DOI: $10.1515 /$ REVCE.2011.002.

Szwast M., 2012. Membrany polimerowe do rozdzielania gazów. Przemyst Chemiczny, 91, 1356-1361.

Szwast M., Fabianowski W., Gradoń L., Piątkiewicz W., 2008. Koncepcja wytwarzania membran kapilarnych oraz metody oceny ich jakości. Przemyst Chemiczny, 87, 206-209.

Szwast M., Lipińska I., Jermakowicz J., Piątkiewicz W., 2007. Modyfikacja własności filtracyjnych i mechanicznych kapilarnych membran polimerowych. II Konferencja Naukowo-Techniczna Doktorantów i Młodych Naukowców. Młodzi naukowcy wobec wyzwań wspótczesnej techniki, Warszawa, 24-26.08.2007, Referaty. 57-63.

Xinli Z., Xiaoling H., Ping G., Guozheng L., 2009. Preparation and pore structure of porous membrane by supercritical fluid. J. Supercrit. Fluids 49, 111 - 116. DOI: 10.1016/j.supflu.2008.09.021.

Yin J.-Z., Wang A.-Q.,Wei W., Liu Y, Shi W-H, 2005. Analysis of the operation conditions for supercritical fluid extraction of seed oil. Sep. Purif. Technol. 43, 2, 163-167. DOI: 10.1016/j.seppur.2004.10.016.

Zhang C.-F., Zhu B.-K., Ji G.-L., Xu Y.-Y., 2007. Supercritical carbon dioxide extraction in membrane formation by thermally induced phase separation. J. Appl. Polymer Sci., 103, 1632-1639. DOI: 10.1002/app.24620.

Received 14 May 2013

Received in revised form 07 July 2013

Accepted 24 July 2013 